\title{
Comparison of Direct Immunofluorescence and Giemsa Staining in Chlamydia trachomatis Follicular Conjunctivitis
}

\author{
Fahimeh Asadi-Amoli, ${ }^{1, *}$ Zohreh Nozarian, ${ }^{1}$ Vahid Mehrtash, ${ }^{2}$ Hooshang Beheshtnejad, ${ }^{3}$ and \\ Avishan Shabani ${ }^{4}$ \\ ${ }^{1}$ Department of Pathology, Tehran University of Medical Sciences, Tehran, IR Iran \\ ${ }^{2}$ Student of Medicine, School of Medicine, Tehran University of Medical Sciences, Tehran, IR Iran \\ ${ }^{3}$ Department of Ophthalmology, Tehran University of Medical Sciences, Tehran, IR Iran \\ ${ }^{4}$ Department of Pharmacology, Tehran University of Medical Sciences, Tehran, IR Iran \\ *Corresponding author: Fahimeh Asadi-Amoli, Department of Pathology, Tehran University of Medical Sciences, Tehran, IR Iran. E-mail: path1383@yahoo.com \\ Received 2011 November 5; Accepted 2011 December 22.
}

\begin{abstract}
Background: Chlamydia trachomatis is the most common sexually transmitted bacterial infection and can cause various types of human eye disease. There are two general methods for diagnosis of chlamydial infection; cell culture and non-culture tests.

Objectives: In this study we evaluated the prevalence of $C$. trachomatis in follicular conjunctivitis and compared the two rapid direct methods [direct immunofluorescence(DIF) and Giemsa staining] used in diagnosis of chlamydial conjunctivitis.

Patients and Methods: In this descriptive and comparative study, 285 patients with follicular conjunctivitis were recruited and admitted to the referral laboratory unit of Farabi Eye Hospital of Tehran and 570 conjunctival scrapings from their right and left eyes were collected and subsequently stained via DIF and Giemsa staining.

Results: In this study, 109 (50 males and 59 females) patients (38.24\%) had positive results with DIF technique and most of these patients aged 21 - 30 and 11 - 20 years respectively. A number of 47 patients (27 males and 20 females) (16.49\%) had positive results with Giemsa staining and in compared to DIF, sensitivity and specificity of Giemsa method was $38.53 \%$ and $97.16 \%$ respectively.

Conclusions: Compared with Giemsa staining, DIF is a suitable test with high sensitivity and specificity and the choice test for rapid diagnosis of chlamydial conjunctivitis for routine purposes.
\end{abstract}

Keywords: Follicular Conjunctivitis, Direct Immunofluorescence, Giemsa Staining, Chlamydia trachomatis

\section{Background}

Chlamydia is a genus of bacteria that consists of intracellular obligate parasites and its members fall into one of these three species: Chlamydia trachomatis, Chlamydia psittaci or Chlamydia pneumoniae. This organism can present itself in two different forms during its life-cycle; elementary body (EB) which is the infectious form and reticulate body (RB), the non-infectious form. The EB obtains a protein component termed major outer membrane protein (MOMP) which is specific for each species and sub-species and can be identified by a monoclonal antibody (Ab). C. trachomatis is divided into 18 serotypes. Serotypes A, B, Ba and C can cause hyper-endemic blinding trachoma in humans and serotypes D, E, F, G, H, I, J and K are responsible for chlamydia-related pneumonia in neonates and chlamydia-related inclusion conjunctivitis, non-gonococcal urethritis, cervicitis, salpingitis, pelvic inflammatory disease, proctitis and epididymitis in infants and adults. Serotypes L1, L2 and L3 are the perpetrators of lymphogranuloma venereum [1].

Gallo Vaulet et al. conducted a study in which the distribution of $C$. trachomatis genotypes among symptomatic patients was evaluated. The researchers found an association between genotype E and neonatal conjunctivitis [2].
Chlamydial infections are one of the most common eye diseases in humans and can cause different types of eye infections including neonatal inclusion conjunctivitis, adult chlamydial keratoconjunctivitis and trachoma. The inflammation of conjunctival stroma in chronic conjunctivitis is presented via formation of papillae and follicles. Chronic follicular conjunctivitis or a type of conjunctivitis that lasts longer than 16 days and is associated with follicle formation in conjunctiva can be related to different etiologies which are either of infectious or non-infectious origin. Chlamydial infections are one of the infectious etiologies of chronic follicular conjunctivitis and this specific association is the area of interest in our research [3,4]. Laboratory diagnostic techniques used for chlamydial infections are either culture-based (cell culture) or nonculture based methods. The culture method is the reference and gold standard method for chlamydia identification. The non-culture based assays for chlamydia detection are as follows: 1- Direct testing like detection of inclusion bodies via Giemsa staining and detection of chlamydia EBs by immunofluorescence staining (DFA; direct fluorescent antibody) 2- Serologic tests such as complement fixation, enzyme-

Copyright (C) 2015, Zahedan University of Medical Sciences. This is an open-access article distributed under the terms of the Creative Commons Attribution-NonCommercial 4.0 International License (http://creativecommons.org/licenses/by-nc/4.0/) which permits copy and redistribute the material just in noncommercial usages, provided the original work is properly cited. 
linked immunosorbent assay (ELISA), indirect immunofluorescence assay (IFA) and microimmunofluorescence (MIF).3Molecular tests including polymerase chain reaction (PCR), ligase chain reaction (LCR) and DNA-RNA hybridization $[1,5]$.

\section{Objectives}

In this study we aimed to assess the prevalence of C. trachomatis in conjunctival specimens of patients diagnosed with follicular conjunctivitis and also compare the two direct testing techniques implemented in chlamydial conjunctivitis diagnosis, i.e. Giemsa staining and direct immunofluorescence (DIF).

\section{Patients and Methods}

In this descriptive and comparative study, 285 patients primarily diagnosed with follicular conjunctivitis and also clinically suspected to be infected with C. trachomatis were referred to the clinical laboratory of Farabi Eye Hospital for diagnosis of C. trachomatis infection. For all subjects in this study, the conjunctival scraping method was applied in order to obtain conjunctival specimens. For this purpose, the tarsal conjunctiva of upper and lower eyelids of both right and left eyes was scraped in a sterile fashion. Subsequently, the specimens were separately placed on regular microscopic glass slides and immunofluorescence slides and stained via the two Giemsa and DIF methods. In the Giemsa method samples were stained with Giemsa stain after air-drying and in the DIF method slides were first methanol-fixed for five minutes and then stained with a monoclonal IgG Ab conjugated with fluorescein that detects the MOMP of all 18 serotypes of $C$. trachomatis (Product name: C. trachomatis DFA kit; Code:PL.1010; Manufacturer: Pro-Lab Diagnostics, Canada). The Giemsa-stained and DIF-stained slides were viewed and evaluated under regular optical microscope and immunofluorescence microscope, respectively. Samples that were not sufficient enough in terms of adequate number of epithelial cells, etc. were excluded from the study and re-sampling was performed in these cases. In this study patients were not charged with any additional expense and as they referred to the lab for diagnostic purposes, routine sampling was carried out. It should be noted that no additional intervention was carried out in this study and standard sampling and staining for diagnosis of conjunctival lesions was performed upon physician request. The results of the two staining methods were recorded and the related data was statistically evaluated. In order to compare the two diagnostic methods put to use in this study, we considered the DIF assay as the standard technique in this scientific endeavor (C. trachomatis infection is confirmed when DIF is positive). Sensitivity, specificity, false positive rate and false negative rate of the Giemsa staining method were extracted. For calculating these four values, we used the following formulas:

Sensitivity $=$ true positive/(true positive + false negative), Specificity $=$ true negative $/($ true negative + false positive $)$,
False positive rate $=1$ - specificity $=$ false positive $/$ (false positive + true negative),

False negative rate $=1$ - sensitivity $=$ false negative $/($ false negative + true positive)

Table 1. Results of Comparison of DIF and Giemsa Staining Techniques Based on Different Age Groups ${ }^{\mathrm{a}}$

\begin{tabular}{lccccc}
\hline Age, $\mathbf{y}$ & DIF+ Giemsa+ & $\begin{array}{c}\text { DIF+, } \\
\text { Giemsa+ }\end{array}$ & $\begin{array}{c}\text { DIF+, } \\
\text { Giemsa- }\end{array}$ & $\begin{array}{c}\text { DIF-, } \\
\text { Giemsa+ }\end{array}$ \\
\hline$<\mathbf{1}$ & & & & & \\
Male & 1 & 1 & 1 & 0 & 0 \\
Female & 0 & 0 & 0 & 0 & 0 \\
1- 10 & & & & & \\
Male & 2 & 0 & 0 & 2 & 0 \\
Female & 4 & 1 & 1 & 3 & 0
\end{tabular}

$11-20$

\begin{tabular}{cccccc} 
Male & 9 & 9 & 7 & 2 & 2 \\
\hline Female & 12 & 3 & 3 & 9 & 0 \\
\hline $\mathbf{2 1 - 3 0}$ & & & & & \\
Male & 26 & 11 & 10 & 16 & 1 \\
Female & 14 & 5 & 5 & 9 & 0 \\
\hline
\end{tabular}

31 - 40

\begin{tabular}{|cccccc} 
Male & 3 & 1 & 1 & 2 & 0 \\
\hline Female & 12 & 4 & 4 & 8 & 0 \\
\hline $\mathbf{4 1 - 5 0}$ & & & & & \\
\hline Male & 5 & 2 & 2 & 3 & 0 \\
\hline Female & 11 & 6 & 6 & 5 & 0 \\
\hline
\end{tabular}

\section{1- 60}

\begin{tabular}{cccccc} 
Male & 3 & 1 & 1 & 2 & 0 \\
\hline Female & 4 & 1 & 1 & 3 & 0 \\
\hline $\begin{array}{l}\text { 61-70 } \\
\text { Male }\end{array}$ & 0 & 1 & 0 & 0 & 1 \\
\hline Female & 1 & 0 & 0 & 1 & 0 \\
\hline 70 & & & & & \\
\hline Male & 1 & 1 & 0 & 1 & 1 \\
\hline Female & 1 & 0 & 0 & 1 & 0 \\
\hline $\begin{array}{l}\text { All age } \\
\text { groups }\end{array}$ & & & & & \\
\hline Male & 50 & 27 & 22 & 28 & 5 \\
\hline Female & 59 & 20 & 20 & 39 & 0 \\
\hline
\end{tabular}

a Abbreviation: DIF: direct immunofluorescence. 
Asadi-Amoli F et al.

\section{Results}

A total of 285 follicular conjunctivitis patients suspected to have a chlamydial infection underwent sampling from right and left tarsal conjunctivae. A number of 109 subjects (38.24\%) were positive for C. trachomatis when DIF was implemented (Figure 1). To be noted, 50 of them were males (45.7\%) and 59 of them females (54.1\%). Most of the patients who tested positive with DIF were in their third and second decades of life (21 - 30 and 11 - 20 years), with prevalence of $36.69 \%$ and $19.26 \%$ respectively.

On the other hand, a total number of 47 patients (16.49\%) (27 males [57.44\%] and 20 females [42.55\%]) showed positive results for $C$. trachomatis when the Giemsa staining method was used (Figure 2). A more detailed depiction of the results which is based on different age groups is demonstrated in Table 1.

The false negative and false positive rates of Giemsa staining were $61.47 \%$ and $2.84 \%$, in order. Taking DIF method into account, sensitivity, and specificity of Giemsa method equaled $38.53 \%$ and $97.16 \%$, respectively

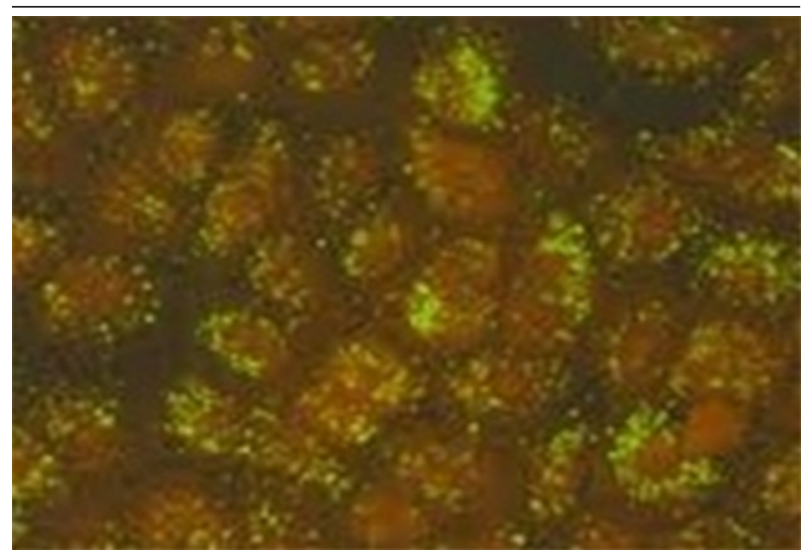

Figure 1. Epithelial cells of conjunctiva containing intra-cytoplasmic inclusions labeled with fluorescent materials (yellow dots), direct immunofluorescence method, fluorescence microscope, $100 \times$ magnification.

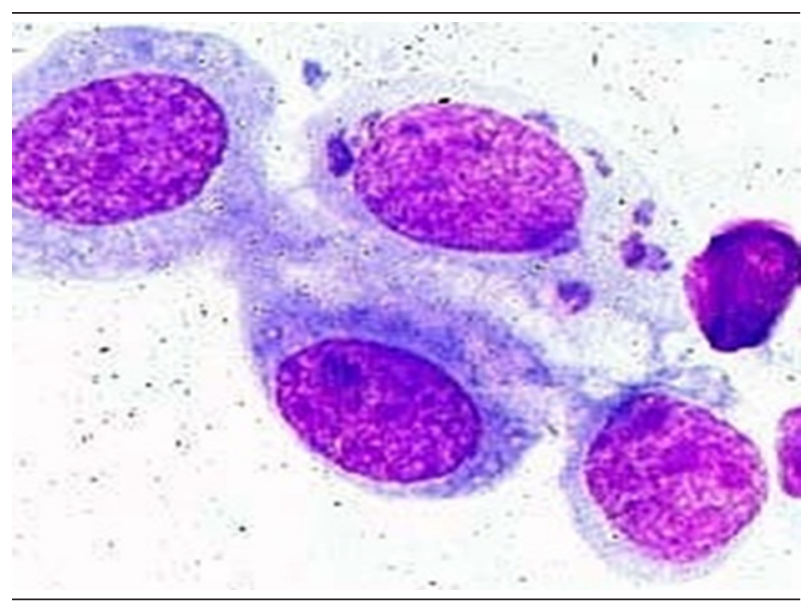

Figure 2. Epithelial cells of conjunctiva containing intra-cytoplasmic inclusions "draped" around nucleus, Giemsa staining method, optical microscope, $400 \times$ magnification.

\section{Discussion}

Among the two staining procedures used in this study, the DIF method was chosen as the standard technique; therefore, the Giemsa method faced false negative and false positive rates of $61.47 \%$ and $2.84 \%$, respectively. In comparison to DIF, Giemsa staining's sensitivity and specificity was $38.53 \%$ and $97.16 \%$, in order. C. trachomatis is the most common sexually transmitted bacterial infection (STI) [6, 7] and its prevalence differs with age, gender, race, and residency of the infected person and type of diagnostic test [6]. Chlamydial infections are one of the most frequent ophthalmologic disorders and a study declared that among 15 - 44 year olds, 1 out of every 44,000 of them contract the infection each year and prevalence in total population was 1 in 100000 [8]. Several studies have pointed out C. trachomatis as the most common etiology of chronic follicular conjunctivitis and most significant cause of bacterial conjunctivitis in neonates [9]. However, the prevalence of this infection has varied from 20 - 90\% in scientific literature, which is parallel to age groups and the prevalence of chlamydial eye infections positively correlates with genital disease prevalence $[5,8]$

In our study most of the patients belonged to the 21 30 years age group and after that, 11 - 20 years age group. After the age of 50, a significant decrease in the number of positive cases was witnessed in our study and this in accord with similar studies [10-13]. Our study was mainly based on adult chronic follicular conjunctivitis and we only had one pediatric case aged less than one year because neonates usually receive treatment in child therapeutic centers and are less frequently referred to ophthalmologic hospitals. In our country, prior to our current effort, only one study has been carried out to evaluate the association between chlamydia and conjunctivitis but that assessment was limited to the neonatal age group and considered neonatal conjunctivitis. In that specific study, specimens from 170 neonates diagnosed with conjunctivitis were obtained and 10 samples (6\%) tested positive for chlamydia via DIF method. The relatively low prevalence of chlamydial conjunctivitis in the neonates of that study, compared to reports from the West, was linked to a lower prevalence of STIs in Iran [10]. Regarding neonatal conjunctivitis, a study in Argentina reported a $7.8 \%$ prevalence of chlamydia-related conjunctivitis [11]. In our study from the total 285 patients referred to the lab, 109 of them (38.24\%) were positive in terms of chlamydial infection. Similar studies have reported positive results of $8.6 \%$ and $38 \%$ in sample sizes of 93 and 100 patients, respectively $[12,13]$. It should be noted that neither our study nor others' detected a significant sex-based difference in chlamydial conjunctivitis contraction $[2,9,11]$.

Considering the age distribution in this assessment and the fact that most of the patients were part of the more sexually active age group and even some of them had previously experienced genitourinary infections, spread of infection through genital means and by eye-hand con- 
tact can be an important factor in disease transmission. Based on patients' medical history, other factors related to disease transmission are polluted water bodies, residing in sub-urban areas, swimming pools and sharing nonhygienic cosmetic agents (especially common in young women).Various methods are available for the laboratory diagnosis of infections caused by C. trachomatis. These assays differ in sensitivity, specificity and positive predictive value (PPV). The most suitable test or a combination of different diagnostic evaluations is determined based on several factors: 1- the susceptible population 2- accessible facilities 3-price of consumables 4- technician's skill 5- type of specimen [14]. Prevalence and virulence of the disease in the tested population are the most important criteria for type of test. The type of the selected sample depends on the symptoms of the disease. Generally, laboratory diagnosis of chlamydial infections is based on culture and non-culture methods.

Cell culture is the gold standard and reference method for diagnosis of chlamydial infections and must be carried out in suspicious cases. Sensitivity, specificity and PPV of the culture method vary in different reports. Based on these reports, theses have ranged 50 - 95\% for sensitivity, $92-100 \%$ for specificity and $73-98 \%$ for PPV [14]. The problems associated with performing cell culture are: 1 - it requires a specific transport medium, 2 - cells need to be stored at four degrees centigrade, 3- specimens must be cultured by a maximum 4 - 6 hours after sampling, 4- it is a time-consuming and expensive method and 5- it is inaccessible at most labs, especially in regions where chlamydia is not endemic. Therefore, this method is currently not used as a main diagnostic technique and is especially not used when samples are recruited non-invasively [1517]. Recent articles and reports indicate that the sensitivity of cell culture is not higher than that of other methods such as DFA, enzyme immunoassay (EIA) and nucleic acid probe (NAP)-based technique and for ophthalmologic samples; these methods are more preferred compared to other methods [15]. Another method used for diagnosis of $C$. trachomatis, especially in under-equipped centers, is the direct technique of smear preparation and staining via Giemsa method in order to view intra-cytoplasmic inclusions. This technique is especially useful in neonates and obtains a lower sensitivity for adults. On the other hand, it is easy to perform and is accessible. Ultimately, this technique has established itself as one of the primary, well-known methods in our country, even though it does not have high sensitivity.

A serologic technique is less useful in diagnosing trachoma and inclusion bodies but is the test of choice for neonatal infections and diagnosing pneumonia caused by $C$. trachomatis in children. In general, serologic methods face some limitations due to low sensitivity and specificity and should be confirmed by other assays $[7,12]$ because in many individuals the antibodies that are found are related to a previous infection and because chlamydial infections are mostly localized, a 4-fold increase in $\mathrm{Ab}$ titer is not useful for diagnosis. Also, the Ab titer may not rise until two weeks and this could cause an inacceptable delay in diagnosis of the disease. However, with advances in serologic methods and using specific responses to specific groups of C. trachomatis antigens via MIF method, it is possible to detect a novel infection in patients who were previously infected with another immunotype [15]. Using non-culture and non-amplified techniques such as DNAprobe test is specifically reserved for situations where PCR and culture methods are not available [18]. Nucleic acid amplification tests (NAATs) e.g. PCR obtain the highest sensitivity and their specificity is approximately equal to cell culture. Due to its high sensitivity and high specificity, NAAT is more effective for non-invasive samples. However, using this technique for routine diagnostic purposes regarding eye infections is costly and the technique is mostly used in research $[19,20]$. DIF technique is the most suitable method for diagnosing chlamydial conjunctivitis. This rapid test has a relatively high sensitivity (70-100\%) compared to cell culture. The specificity of the test exceeds $95 \%$ ( 87 - 99\%). It is the only test that allows evaluation of specimen adequacy. Samples containing 10 - 20 columnar and/or squamous metaplastic cells are acceptable. Antibodies against MOMP in chlamydia are species-specific. This technique is very suitable for routine diagnostic means [15].

Taking the aforementioned characteristics of DIF and its high sensitivity and high specificity into account, we considered the DIF method as the standard test of choice in our study and the other diagnostic test used in our study, i.e. Giemsa staining (which is the current method used in most labs) was compared to it. A few similar studies have been conducted in this area (comparing different nonculture methods, especially the two direct testing techniques, DIF and Giemsa staining) and most of them have evaluated women's vaginal samples. In a study carried out by Palayekar et al. the two direct tests, i.e. DIF and Giemsa staining were compared to each other. In total, $16.8 \%$ and $10 \%$ of the samples were DIF-positive and Giemsa-positive, respectively [21]. In another study, the sensitivity of DIF and Giemsa method for identifying C. trachomatis inclusions in McCoy cells was assessed. The results showed higher sensitivity of DIF in comparison to Giemsa [22]. In a study performed by Lin et al. [13], rapid diagnostic methods for chlamydial conjunctivitis were examined. Of the total conjunctival scraping samples, 38 of them stained positive with DIF and 29 of them stained positive with Giemsa. The DIF method consumed five more minutes of time compared to Giemsa staining (45 minutes vs. 40 minutes). On the other hand, detecting a positive result in DIF was less difficult compared to detecting one in Giemsa method [13]. In a completely accidental manner (3\% of all cases), simultaneous incidence of chlamydia and adenovirus infection is evident and this finding should be anticipated in patients with long-standing follicular keratoconjunctivitis [23]. In conclusion, the Giemsa staining technique is a method with low sensitivity (38.53\%) 
and high specificity (97.16\%) in comparison to DIF. Therefore, Giemsa method is not suitable for routine diagnostic means considering chlamydial conjunctivitis and the possibility of not diagnosing numerous patients infected with C. trachomatis is increased when using this assay. Regarding its ease of performance and high specificity and high sensitivity, DIF is a suitable technique and the method of choice for diagnosis of chlamydial conjunctivitis. However, for a more accurate and more efficient assessment of the sensitivity and specificity of this technique, a parallel study comparing DIF with techniques such as culture and PCR is recommended.

\section{Acknowledgments}

We would like to extend our gratitude to the pathology laboratory staff of Farabi Hospital, especially Mrs. Nezamabady and Mrs. Sharifinejad, for their effective cooperation and technical assistance. Of note, this study was not affiliated to a thesis or research proposal and was solely conducted in the Farabi Hospital laboratory.

\section{Footnotes}

Authors' Contribution:Fahimeh Asadi-Amoli and Zohreh Nozarian: Obtaining samples, observation of pathology slides, interpretation of results, drafting the manuscript. Vahid Mehrtash: Translation and language editing. Hooshang Beheshtnejad: Clinical diagnosis and lab patient referral. Avishan Shabani: Statistical calculations.

Funding/Support:Tehran University of Medical Sciences.

\section{References}

1. Mahon CR, Lehman DC, Manuselis G. Textbook of diagnostic microbiology. 4 th ed. Philadelphia:W.B. Saunders; 2010. pp. 551-6.

2. Gallo Vaulet L, Entrocassi C, Corominas AI, Rodriguez Fermepin M. Distribution study of Chlamydia trachomatis genotypes in symptomatic patients in Buenos Aires, Argentina: association between genotype E and neonatal conjunctivitis. BMC Res Notes. 2010;3:34.

3. Spenser WH. Ophthalmic pathology: An atlas and textbook. Philadelphia: W.B. Saunders; 1996. pp. 71-3.

4. Yanoff M, Sassani JW. Ocular pathology. 4 th ed. Philadelphia: W.B. Saunders; 2014. pp. 231-2.

5. Tille PM. Bailey\& Scott diagnostic microbiology. 13 th ed. USA: Mosby; 2013. pp. 515-9.

6. Joesoef MR, Weinstock HS, Johnson RE. Factors associated with recurrent chlamydial infection and failure to return for retesting in young women entering national job training program, 1998-2005. Sex Transm Dis. 2008;35(4):368-71.

7. Meyer T. [Modern diagnosis of Chlamydia trachomatis infections]. Hautarzt. 2007;58(1):24-30.

8. Tullo AB, Richmond SJ, Easty DL. The presentation and incidence of paratrachoma in adults. J Hyg (Lond). 1981;87(1):63-9.

9. Rours IG, Hammerschlag MR, Ott A, De Faber TJ, Verbrugh HA, de Groot R, et al. Chlamydia trachomatis as a cause of neonatal conjunctivitis in Dutch infants. Pediatrics. 2008;121(2):e321-6.

10. Soltanzadeh MH, editor. Epidemiological study of neonatal conjunctivitis.; Proceeding of the 24th international congress of pediatrics.; 2004; Cancun, Mexico.

11. Di Bartolomeo S, Mirta DH, Janer M, Fermepin MR, Sauka D, Magarinos F, et al. Incidence of Chlamydia trachomatis and other potential pathogens in neonatal conjunctivitis. Int J Infect Dis. 2001;5(3):139-43.

12. Haller EM, Auer-Grumbach P, Stuenzner D, Kessler HH, Pierer K, Zenz $\mathrm{H}$, et al. Evaluation of two nonculture antigen tests and three serotests for detection of anti-chlamydial antibodies in the diagnosis of ocular chlamydial infections. Graefes Arch Clin Exp Ophthalmol.1996;234(8):510-4.

13. Lin J, Li Y, Zhang J, Feng G, Zhang P, Zheng H, et al. Rapid diagnosis of chlamydial conjunctivitis in laboratory. Yan Ke Xue Bao. 1999;15(3):191-4.

14. Bashour M. Ophthalmologic manifestations of Chlamydia. 2014. Available from: http://reference.medscape.com/article/1203385-overview.

15. Mcpherso RA, Pincus MR. Henrys clinical diagnosis and management by laboratory methods. 22th ed. Philadelphia: W.B. Saunders; 2012. pp. 1066-9.

16. Chandran L, Boykan R. Chlamydial infections in children and adolescents. Pediatr Rev. 2009;30(7):243-50.

17. Watson EJ, Templeton A, Russell I, Paavonen J, Mardh PA, Stary A, et al. The accuracy and efficacy of screening tests for Chlamydia trachomatis: a systematic review. J Med Microbiol. 2002;51(12):1021-31.

18. Olshen E, Shrier LA. Diagnostic tests for chlamydial and gonorrheal infections. Semin Pediatr Infect Dis. 2005;16(3):192-8.

19. Javaloy J, Ferrer C, Vidal MT, Alio JL. Follicular conjunctivitis caused by Chlamydia trachomatis in an infant Saharan population: molecular and clinical diagnosis. Br J Ophthalmol. 2003;87(2):142-6.

20. Taylor-Robinson D, Thomas BJ. Laboratory techniques for the diagnosis of chlamydial infections. Genitourin Med.1991;67(3):256-66.

21. Palayekar VV, Joshi JV, Hazari KT, Shah RS, Chitlange SM. Comparison of four nonculture diagnostic tests for Chlamydia trachomatis infection. J Assoc Physicians India. 2000;48(5):481-3.

22. Munday PE, Johnson AP, Thomas BJ, Taylor-Robinson D. A comparison of the sensitivity of immunofluorescence and Giemsa for staining Chlamydia trachomatis inclusions in cycloheximide-treated McCoy cells. J Clin Pathol.1980;33(2):177-9.

23. Mellman-Rubin TL, Kowalski RP, Uhrin M, Gordon YJ. Incidence of adenoviral and chlamydial coinfection in acute follicular conjunctivitis. Am J Ophthalmol. 1995;119(5):652-4. 